\title{
Exotic states at the LHC
}

\author{
Liming Zhang ${ }^{* \dagger}$ \\ Center for High Energy Physics, Tsinghua University, Beijing, China \\ E-mail: liming.zhang@cern.ch
}

Using the Run-I data delivered by the LHC, several exotic states are observed or searched for. The most recent $\mathrm{LHCb}$ results are reported, including the search for the $B_{s}^{0} \pi^{ \pm}$state, the observation of four $J / \psi \phi$ structures in $B^{+} \rightarrow J / \psi \phi K^{+}$decays, and the observation of $J / \psi p$ resonances consistent with pentaquark states in $\Lambda_{b}^{0} \rightarrow J / \psi p K^{-}$decays. The results on $J / \psi \phi$ from the CMS collaboration are also discussed.

38th International Conference on High Energy Physics 3-10 August 2016

Chicago, USA

* Speaker.

${ }^{\dagger}$ on behalf of the LHCb Collaboration 


\section{Introduction}

In 1964 Gell-Mann [1] and Zweig [2] separately proposed that hadrons were formed from fundamental point-like objects now called quarks. The minimal quark configuration can explain all well established hadrons for most of the last half-century. However, in recent decades there have been several observations of mesonic states containing two quarks and two anti-quarks, called tetraquarks [3], and now, as described here, the observation of two pentaquark baryon states [4]. Such multi-quark configurations were also discussed by Gell-Mann [1] and Zweig [2]. Their existence is not forbidden by QCD, as long as they maintain colour-singlet configurations. Here, I discuss the most recent studies on exotic states performed mainly by the LHCb collaboration using the run-I data corresponding to $3 \mathrm{fb}^{-1}$ collected in the years of 2011 and 2012, as well as results from the CMS experiment.

\section{Search for $B_{s}^{0} \pi^{ \pm}$states}

The D0 collaboration reported evidence of a narrow structure, referred to as the $X(5568)$, in the $B_{s}^{0} \pi^{ \pm}$spectrum, with the $B_{s}^{0}$ meson reconstructed by $J / \psi \phi$ [5]. The reported mass and width of this state are $5567.8 \pm 2.9_{-1.9}^{+0.9} \mathrm{MeV}$ and $21.9 \pm 6.4_{-2.5}^{+5.0} \mathrm{MeV}$, respectively. Natural units are used, and whenever two uncertainties are quoted the first is statistical and the second systematic. If this state would be confirmed, it would differ from any of the previous observations, as it must contain constituent quarks with four different flavours $(b, s, u, d)$.

The LHCb experiment has perform a search for such a state [6]. Candidate $B_{s}^{0}$ mesons are reconstructed through $D_{s}^{-} \pi^{-}$, and $J / \psi \phi$. The $B_{s}^{0}$ sample is about 20 times larger than that in the D0. The results of the fit to $B_{s}^{0} \pi^{ \pm}$mass spectrum are shown in Fig. 1 for $p_{\mathrm{T}}\left(B_{s}^{0}\right)>10 \mathrm{GeV}$. The $X(5568)$ yield is not significant for any minimum $p_{\mathrm{T}}\left(B_{s}^{0}\right)$ requirement. The upper limit of the production ratio of $X \rightarrow B_{s}^{0} \pi^{ \pm}$to total $B_{s}^{0}$ for the $X(5568)$ state is set to be $<0.021(0.024)$ in LHCb acceptance for $p_{\mathrm{T}}\left(B_{s}^{0}\right)>10 \mathrm{GeV}$ at $90(95) \%$ confidence level. Furthermore, no significant signal for a $B_{s}^{0} \pi^{ \pm}$resonance is seen at any value of mass below $6 \mathrm{GeV}$ and width, upper limits are set as well.

\section{Observation of four $X \rightarrow J / \psi \phi$ states in $B^{+} \rightarrow J / \psi \phi K^{+}$decays}

Back to 2009, CDF [7] first observed a narrow near threshold $X(4140) \rightarrow J / \psi \phi$ structure in

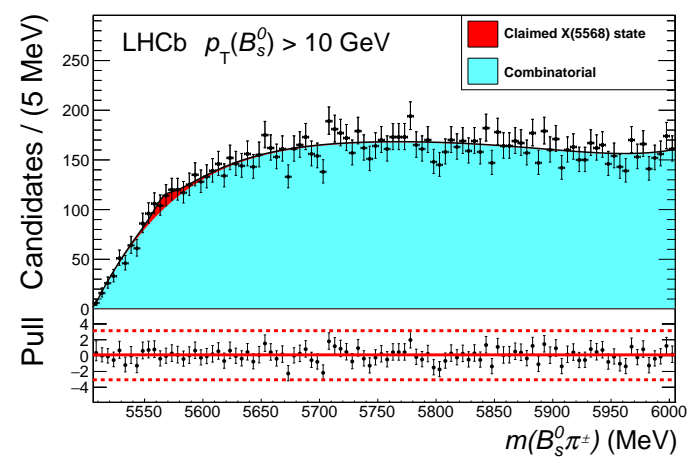

Figure 1: Results of the fit to the $B_{s}^{0} \pi^{ \pm}$mass distribution for candidates with $p_{\mathrm{T}}\left(B_{s}^{0}\right)>10 \mathrm{GeV}$. 


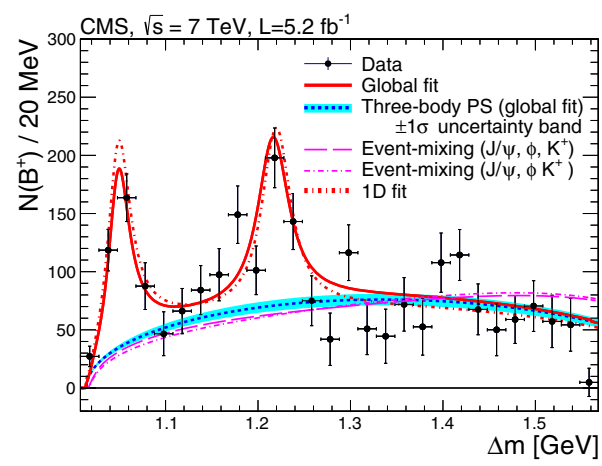

Figure 2: The number of $B^{+} \rightarrow J / \psi \phi K^{+}$candidates as a function of $\Delta m=m\left(\mu^{+} \mu^{-} K^{+} K^{-}\right)-m\left(\mu^{+} \mu^{-}\right)$.

$B^{+} \rightarrow J / \psi \phi K^{+}$decays, with $M=4143.4 \pm 3.0 \pm 0.6 \mathrm{MeV}$, and $\Gamma=15.3_{-6.1}^{+10.4} \pm 2.5 \mathrm{MeV}$. The structure was not confirmed by the Belle [8], Babar [9], and early low statistic LHCb [10] data, however, observed by the CMS [11] and D0 [12] collaborations. There is also an evidence for a second structure $X(4274)$ in the CDF and CMS data. The CMS distribution is shown in Fig. 2 after subtracting a very large combinatorial background. $2480 \pm 160$ signal events are retained.

$\mathrm{LHCb}$ [13] has performed the first amplitude analysis of $B^{+} \rightarrow J / \psi \phi K^{+}$decays, investigating the $J / \psi \phi$ structures. The number of signal events is $4289 \pm 141$ with a background fraction of $23 \%$ accounted for the amplitude analysis. The data can be described including seven $K^{*+} \rightarrow \phi K^{+}$ resonances, with floated masses and widths, four $X \rightarrow J / \psi \phi$ structures, and non-resonant $\phi K^{+}$and $J / \psi \phi$ contributions. The mass projections of $\phi K^{+}$and $J / \psi \phi$ are shown in Fig. 3. Table 1 shows the results for the $X$ structures, including the determined masses, widths, fit fractions and $J^{P}$ 's. The four $X$ structures and a non-resonant $J / \psi \phi$ contribution all have significance above $5 \sigma$. The existence of $X(4274)$ is established. The quantum numbers of $X(4140)$ and $X(4274)$ states are identified as $1^{++}$for $>5 \sigma$ significance, while the $X(4500)$ and $X(4700)$ states as $0^{++}$for $>4 \sigma$. Notably, the $X(4140)$ width is substantially larger than previously determined.
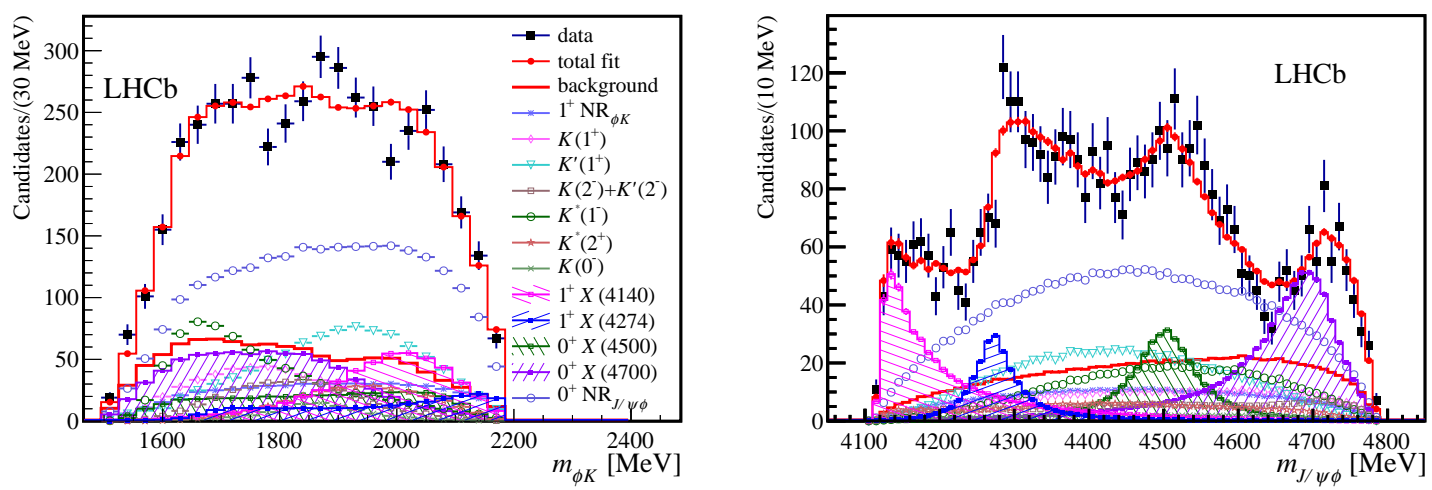

Figure 3: Distributions of $\phi K^{+}$(left) and $J / \psi \phi$ (right) invariant masses for the $B^{+} \rightarrow J / \psi \phi K^{+}$candidates (black data points) compared with the results of the default amplitude fit.

\section{Observation of $J / \psi p$ states consistent with pentaquark in $\Lambda_{b}^{0} \rightarrow J / \psi p K^{-}$decays}

The decay $\Lambda_{b}^{0} \rightarrow J / \psi p K^{-}$was first observed by LHCb with unexpected large yield and has 
Table 1: Results for significances, masses, widths and fit fractions (FF) of the components.

\begin{tabular}{cclcr} 
Resonance & Significance & $M_{0}[\mathrm{MeV}]$ & $\Gamma_{0}[\mathrm{MeV}]$ & FF $\%$ \\
\hline \hline$X(4140)$ & $8.4 \sigma$ & $4146.5 \pm 4.5_{-2.8}^{+4.6}$ & $83 \pm 21_{-14}^{+21}$ & $13 \pm 3.2_{-2.0}^{+4.7}$ \\
$X(4274)$ & $6.0 \sigma$ & $4273.3 \pm 8.3_{-3.6}^{+17.2}$ & $56 \pm 11_{-11}^{+8}$ & $7.1 \pm 2.5_{-2.4}^{+3.5}$ \\
$X(4500)$ & $6.1 \sigma$ & $4506 \pm 11_{-15}^{+12}$ & $92 \pm 21_{-20}^{+21}$ & $6.6 \pm 2.4_{-2.3}^{+3.5}$ \\
$X(4700)$ & $5.6 \sigma$ & $4704 \pm 10_{-24}^{+14}$ & $120 \pm 31_{-33}^{+42}$ & $12 \pm 5_{-5}^{+9}$ \\
$\mathrm{NR}_{J / \psi \phi}$ & $6.4 \sigma$ & & & $46 \pm 11_{-21}^{+11}$
\end{tabular}

been used to precisely measure the $\Lambda_{b}^{0}$ baryon lifetime [14]. However, one feature of the decay that was not addressed, was an anomalous peaking structure in the $J / \psi p$ invariant mass spectrum, shown in Fig. 4. The peak could correspond to a resonance with minimal quark content of $u u d c \bar{c}$, called pentaquark-charmonium state.

To prove the peak is not due to an interference of various $\Lambda^{*}$ states, $\mathrm{LHCb}$ performed a full amplitude analysis [4]. Firstly, the previous observed conventional resonances $\Lambda^{*}$ and $\Sigma^{*}$ are used to fit the data, but the fit fails to reproduce the structure in $m_{J / \psi p}$. To better describe the data, two $J / \psi p$ states are required. The best fit projections are shown in Fig. 4. Both $m_{K p}$ and the peaking structure in $m_{J / \psi p}$ are reproduced by the fit. The two $P_{c}^{+}$states are found to have masses of $4380 \pm 8 \pm 29 \mathrm{MeV}$ and $4449.8 \pm 1.7 \pm 2.5 \mathrm{MeV}$, with corresponding widths of $205 \pm 18 \pm 86 \mathrm{MeV}$ and $39 \pm 5 \pm 19 \mathrm{MeV}$, and called $P_{c}(4380)^{+}(9 \sigma)$ and $P_{c}(4450)^{+}(12 \sigma)$ [4]. The fractions of the total sample due to the lower mass and higher mass states are $(8.4 \pm 0.7 \pm 4.2) \%$ and $(4.1 \pm 0.5 \pm 1.1) \%$, respectively. The best fit has spin-parity $J^{P}$ values of $\left(3 / 2^{-}, 5 / 2^{+}\right)$for low and high mass states; the other two possibilities $\left(3 / 2^{+}, 5 / 2^{-}\right)$or $\left(5 / 2^{+}, 3 / 2^{-}\right)$aren't ruled out.

However, theoretical models of $\Lambda^{*}$ baryons [15] predict a much larger number of higher mass excitations than is established experimentally, as shown in Fig. 5 (left). Therefore, LHCb inspected the same sample with a model-independent approach for $K^{-} p$ contributions [16]. It's assumed that all $\Lambda_{b}^{0} \rightarrow J / \psi p K^{-}$decays proceed via $\Lambda_{b}^{0} \rightarrow J / \psi \Lambda^{*}, \Lambda^{*} \rightarrow p K^{-}$, with minimal assumptions about the spin and lineshape of possible $\Lambda^{*}$ contributions. This will be referred to as the null-hypothesis $H_{0}$, and examined to see if it describes the data well.
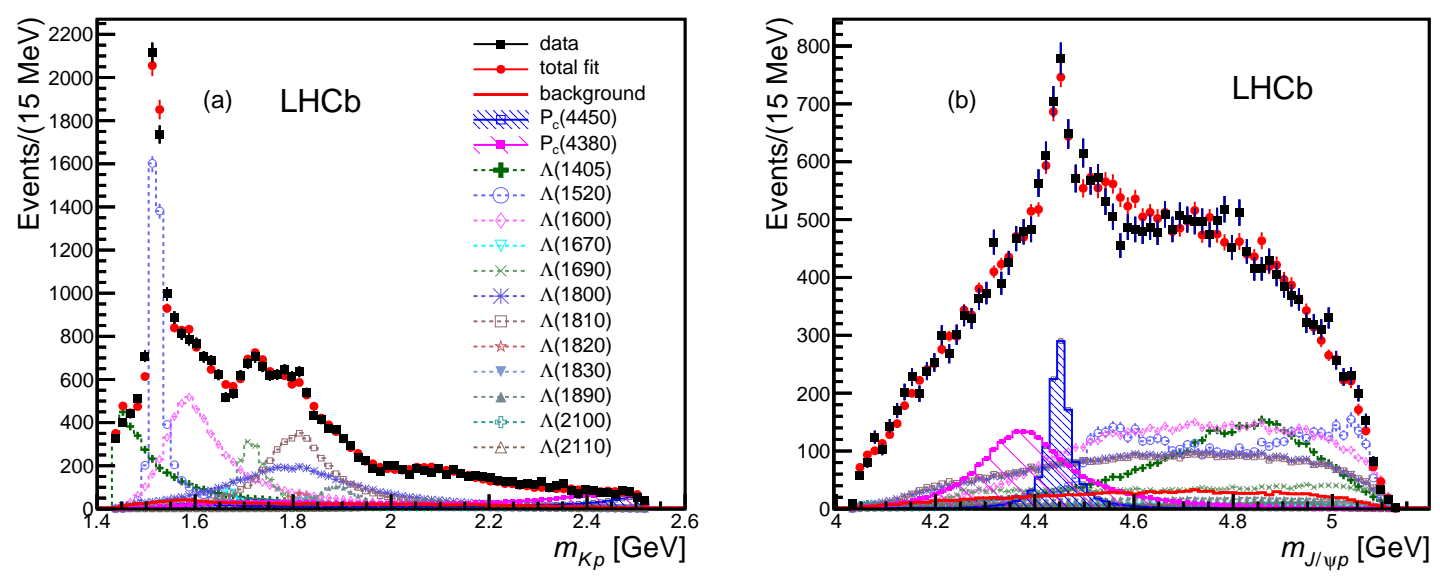

Figure 4: Results of the fit using the reduced $\Lambda^{*}$ model with two $P_{c}^{+}$states. 

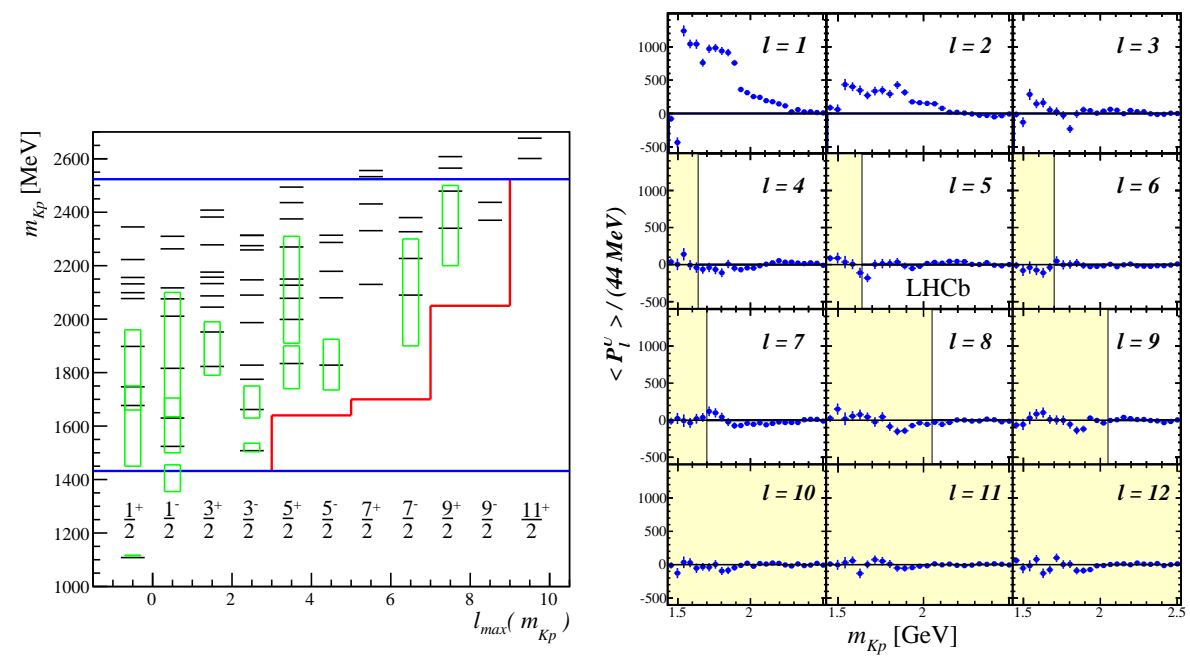

Figure 5: (left) Excitations of the $\Lambda$ baryon. States predicted in Ref. [15] are shown as short horizontal bars (black) and experimentally well-established $\Lambda^{*}$ states are shown as green boxes. Long horizontal lines (blue) shows the range allowed in $\Lambda_{b}^{0} \rightarrow J / \psi p K^{-}$decays. The red stepped line shows the filter for $l_{\max }\left(m_{K p}\right)$. (right) Legendre moments of $\cos \theta_{\Lambda^{*}}$ as a function of $m_{K p}$ in the data. Regions excluded by the $l \leq l_{\max }\left(m_{K p}\right)$ filter are shaded, i.e. the blank region below the red line in the left figure.

The helicity angle distribution $\cos \theta_{\Lambda^{*}}$ can be expanded using Legendre polynomial $P_{l}$

$$
d N / d \cos \theta_{\Lambda^{*}}=\sum_{l=0}^{l_{\max }}\left\langle P_{l}^{U}\right\rangle P_{l}\left(\cos \theta_{\Lambda^{*}}\right)
$$

where $N$ is the efficiency-corrected signal yield, and $\left\langle P_{l}^{U}\right\rangle$ is a Legendre moment of rank $l$. Under the $H_{0}$ hypothesis, $K^{-} p$ components cannot contribute to $l>2 J_{\max }$, where $J_{\max }$ is the highest spin of any $K^{-} p$ contribution. Values of $\left\langle P_{l}^{U}\right\rangle$ as a function of $m_{K p}$ are show in Fig. 5 (right). Generally, higher-spin resonance doesn't contribute to a lower mass. This is used to set $l_{\max }\left(m_{K p}\right)$, displayed in Fig. 5 (left) in the red stepped line. The excluded region are shown in Fig. 5 (right) in shadow. In the region, the values are expected to be zero under the $H_{0}$ hypothesis. Obtained no zero values imply reflections from other channels, $\Lambda_{b}^{0} \rightarrow P_{c}^{+} K^{-}, P_{c}^{+} \rightarrow J / \psi p$ or $\Lambda_{b}^{0} \rightarrow Z_{c}^{-} p, Z_{c}^{-} \rightarrow J / \psi K^{-}$.

To quantify the inconsistency between the $H_{0}$ hypothesis and the data, MC is generated. The resulting $m_{J / \psi p}$ projection is compared with the data distribution in Fig. 6. A likelihood ratio test

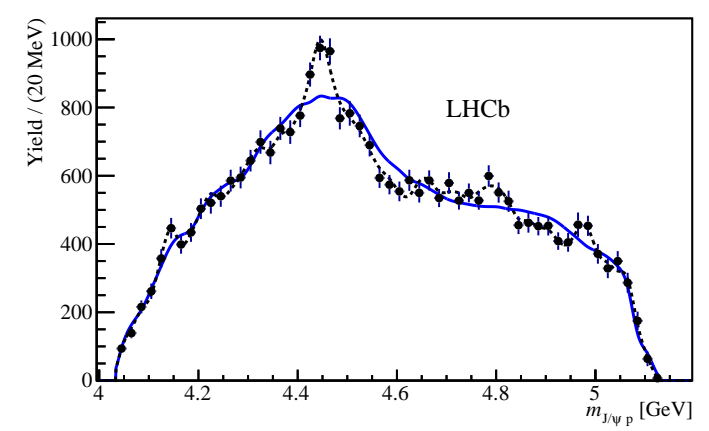

Figure 6: Efficiency-corrected and background-subtracted $m_{J / \psi p}$ distribution of the data (black points with error bars), with PDF obtained from $H_{0}$ (solid blue line) and $\Lambda^{*}$ resonances with spin up to $31 / 2$ (dashed black line) superimposed. 

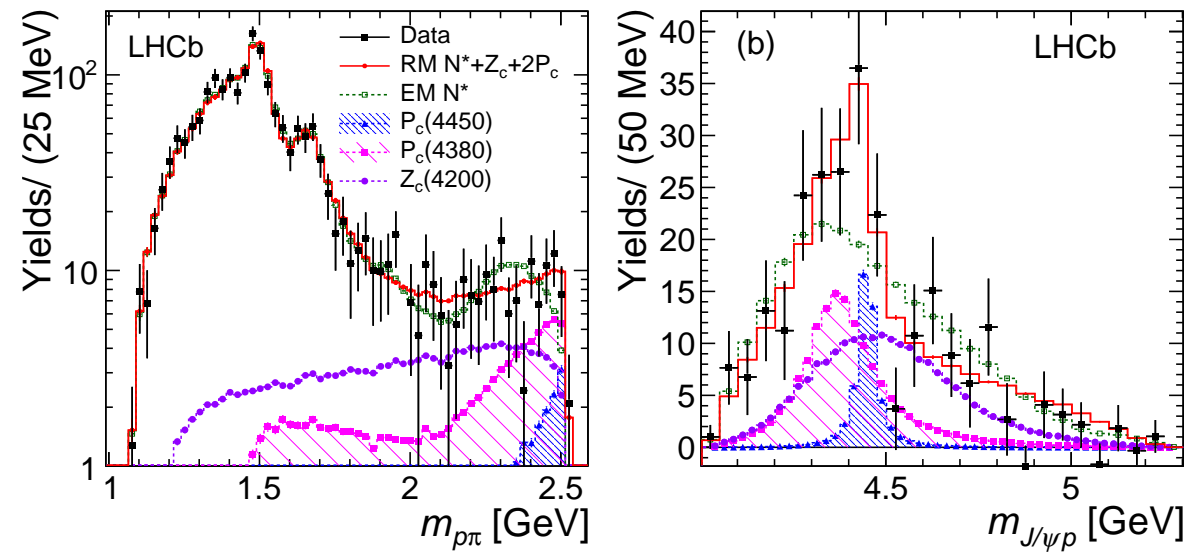

Figure 7: Background-subtracted data and fit projections onto (left) $m_{p \pi}$ and (right) $m_{J / \psi p}$ for the $m_{p \pi}>1.8$ $\mathrm{GeV}$ region. Fits are shown with models containing $14 N^{*}$ states only (called EM) and with reduced $N^{*}$ states (called RM) plus exotic contributions.

shows the $H_{0}$ hypothesis is inconsistent with the data for more than 9 standard deviations. Non$K^{-} p$ contributions, which must be present in the data. This result supports the amplitude modeldependent observation of the $J / \psi p$ resonances presented previously [4]. The model-independent approach can only tell us whether or not exotic state is presents in the data. To obtain the properties of the exotic state if presents, the amplitude model fit is necessary.

\section{Search for exotics states in $\Lambda_{b}^{0} \rightarrow J / \psi p \pi^{-}$decays}

The LHCb collaboration has searched for the observed pentaquark states in $\Lambda_{b}^{0} \rightarrow J / \psi p \pi^{-}$ decays [17], which are related to $\Lambda_{b}^{0} \rightarrow J / \psi p K^{-}$decays via Cabibbo suppression. There are only $1885 \pm 50$ signal events. Amplitude models for the $\Lambda_{b}^{0} \rightarrow J / \psi p \pi^{-}$decays are constructed to examine the possibility of exotic hadron contributions from the $P_{c}(4380)^{+}$and $P_{c}(4450)^{+} \rightarrow J / \psi p$ states and from the $Z_{c}(4200)^{-} \rightarrow J / \psi \pi^{-}$state, previously reported by the Belle collaboration [18] The $N^{*} \rightarrow p \pi^{-}$contributions are expected to dominate. In total $14 N^{*}$ resonances and one $p \pi^{-}$ non-resonant amplitude are used in the model fit.

The fits show a significant improvement when exotic contributions are included. When all three exotic contributions are added to the $14 N^{*}$-only model, their combined statistical significance is $3.1 \sigma$ after including systematic uncertainties. If the $Z_{c}(4200)^{-}$contribution is assumed to be negligible, the two $P_{c}^{+}$states have a significance of $3.3 \sigma$. The exotic contributions can be viewed in the $J / \psi p$ mass projection for $m_{p \pi}>1.8 \mathrm{GeV}$, shown in Fig. 7. The data are found to be consistent with the $P_{c}(4380)^{+}$and $P_{c}(4450)^{+}$production rates expected from their previous observation and Cabibbo suppression [19].

\section{Summary}

In summary, many studies of exotic states have been performed by the LHC experiments at high energy $p p$ collisions. With a sample of $B_{s}^{0}$ mesons much larger than that of D0, the LHCb experiment did not observe any significant $B_{s}^{0} \pi^{ \pm}$signal with a mass below $6 \mathrm{GeV}$. In the full amplitude analysis of the $B^{+} \rightarrow J / \psi \phi K^{-}$decay, four $X \rightarrow J / \psi \phi$ structures have been observed 
by $\mathrm{LHCb}$, among which two of them were seen by the CMS experiment. Two resonant states, $P_{c}(4380)^{+}$and $P_{c}(4450)^{+}$, are observed in the $J / \psi p$ spectrum via a full amplitude analysis of the $\Lambda_{b}^{0} \rightarrow J / \psi p K^{-}$decay. An model independent analysis of this decay confirmed that the data cannot be described without additional exotic states. A full amplitude analysis of the Cabibbosuppressed mode $\Lambda_{b}^{0} \rightarrow J / \psi p \pi^{-}$is also performed, and an evidence of exotic hadron contributions are observed. The results are consistent with those from the Cabibbo-favoured decay.

More studies of exotic hadrons can be made with the LHC run-II data, e.g. in similar channels where $J / \psi$ mesons are replaced by $\psi^{\prime}, \chi_{c 1}, \eta_{c}$ etc. The $\Lambda_{b}^{0} \rightarrow \psi^{\prime} p K^{-}$and $B^{+} \rightarrow \psi^{\prime} \phi K^{+}$decay modes are recently observed by $\mathrm{LHCb}[20]$ and CMS [21] respectively.

\section{References}

[1] M. Gell-Mann, Phys. Lett. 8 (1964) 214.

[2] G. Zweig, CERN-TH-401, 1964.

[3] S. L. Olsen, Front. Phys. (Beijing) 10 (2015) 121, arXiv: 1411 . 7738; A. Pilloni, Nuovo Cim. C39 (2016) 235, arXiv:1508.03823.

[4] LHCb collaboration, R. Aaij et al., Phys. Rev. Lett. 115 (2015) 072001, arXiv : 1507.03414.

[5] D0 collaboration, V. M. Abazov et al., Phys. Rev. Lett. 117 (2016) 022003, arXiv: 1602.07588.

[6] LHCb collaboration, R. Aaij et al., Phys. Rev. Lett. 117 (2016) 152003, arXiv: 1608.00435.

[7] CDF collaboration, T. Aaltonen et al., Phys. Rev. Lett. 102 (2009) 242002, arXiv : 0903.2229 ; CDF collaboration, T. Aaltonen et al., arXiv:1101.6058.

[8] Belle collaboration, S. Cheng-Ping, Chin. Phys. C34 (2010) 615, arXiv: 0912 . 2386.

[9] BaBar collaboration, J. P. Lees et al., Phys. Rev. D91 (2015) 012003, arXiv: 1407.7244.

[10] LHCb collaboration, R. Aaij et al., Phys. Rev. D85 (2012) 091103(R), arXiv: 1202.5087.

[11] CMS collaboration, S. Chatrchyan et al., Phys. Lett. B734 (2014) 261, arXiv:1309. 6920.

[12] D0 collaboration, V. M. Abazov et al., Phys. Rev. D89 (2014) 012004, arXiv: 1309 . 6580 ; D0 collaboration, V. M. Abazov et al., Phys. Rev. Lett. 115 (2015) 232001, arXiv: 1508.07846.

[13] LHCb collaboration, R. Aaij et al., Phys. Rev. Lett. 118 (2016) 022003, arXiv: 1606.07895.

[14] LHCb collaboration, R. Aaij et al., Phys. Lett. B734 (2014) 122, arXiv: 1402 . 62 42; LHCb collaboration, R. Aaij et al., Phys. Rev. Lett. 111 (2013) 102003, arXiv:1307. 2476.

[15] U. Loring, B. C. Metsch, and H. R. Petry, Eur. Phys. J. A10 (2001) 395, arXiv:hep-ph/0103289.

[16] LHCb collaboration, R. Aaij et al., Phys. Rev. Lett. 117 (2016) 082002, arXiv: 1604.05708.

[17] LHCb collaboration, R. Aaij et al., Phys. Rev. Lett. 117 (2016) 082003, arXiv: 1606.06999.

[18] Belle collaboration, K. Chilikin et al., Phys. Rev. D90 (2014) 112009, arXiv: 1408.6457.

[19] H.-Y. Cheng and C.-K. Chua, Phys. Rev. D92 (2015) 096009, arXiv: 1509.03708.

[20] LHCb collaboration, R. Aaij et al., JHEP 05 (2016) 132, arXiv: 1603.06961.

[21] CMS, V. Khachatryan et al., Phys. Lett. B764 (2017) 66, arXiv: 1607.02638. 